\title{
Two-Parameter Harmonic Oscillator Expansion Method for Calculating Non-Relativistic Ground State Energy of Coulomb Three-Particle Systems with Two Identical Particles
}

\author{
Algirdas Deveikis ${ }^{1, \star}$ \\ ${ }^{1}$ Department of Applied Informatics, Vytautas Magnus University, Vileikos 8, LT-44404, Kaunas, Lithuania
}

\begin{abstract}
The variational method in oscillator representation with individual parameters for each Jacobi coordinate is applied to the non-relativistic calculation of the ground state energy of a number of three-particle Coulomb systems, consisting of two identical particles and a different one. The accuracy and convergence rate of the calculations in the constructed oscillator basis are studied up to a total of 28 oscillator quanta. The results are compared with those of the traditional approach using only one such nonlinear variational parameter. The method with individual parameters for Jacobi coordinates is found to possess a number of advantages as compared to the traditional approach.
\end{abstract}

\section{Introduction}

The variational method in the oscillator representation has a number of significant advantages [1] and is widely used in quantum physics for calculating the properties of quantum systems. However, for long-range potentials of Coulomb type the method of harmonic oscillator expansion is known to show a relatively low rate of convergence. This circumstance considerably complicates the application of this method to the calculation of systems with realistic potentials. In Ref. [2], a new variational method is proposed for calculating the energy spectrum of three-particle Coulomb systems, consisting of two identical particles and a different one. In this method, individual parameters for each Jacobi coordinate are introduced instead of only one such parameter in the traditional approach. In the referred paper, this method was applied to calculate the ground state energy of a number of systems in the oscillator basis with zero number of oscillator quanta. In Ref. [3] the method using the oscillator basis with individual parameters for Jacobi coordinates was developed for the non-relativistic calculation of the ground state energy of a Coulomb system consisting of three different particles. For a nonzero number of oscillator quanta it showed much higher accuracy and convergence rate than the calculation with one nonlinear variational parameter. Keeping in mind the advantages of introducing individual parameters for Jacobi coordinates in the case of systems of three different particles, in the present work we apply the method of [2] to the calculation of the ground state energy for a number of Coulomb systems, comprising two identical particles and a different one at nonzero numbers of oscillator quanta and report results of the comparison of its accuracy and convergence rate with those of the traditional calculation. Thanks to the availability of practically "exact" solutions for three-particle Coulomb

\footnotetext{
^e-mail: algirdas.deveikis@vdu.lt
} 
systems [4-9], it appears possible to perform an actually rigorous evaluation of the advantage of the method [2] over the traditional approach.

\section{Hamiltonian and harmonic oscillator expansion method}

The non-relativistic intrinsic Hamiltonian for the three-particle bound-state system with two identical particles and a different one coupled by Coulomb interaction can be written as [2]

$$
\frac{\hat{H}_{\text {intr. }}\left(\boldsymbol{\rho}_{1}, \boldsymbol{\rho}_{2}\right)}{v_{2} c^{2}}=-\frac{1}{2} v_{12} \frac{1}{\gamma_{1}^{2}} \Delta_{\rho_{1}}-\frac{1}{2} \frac{1}{\gamma_{2}^{2}} \Delta_{\rho_{2}}+\frac{2 Z_{1} Z_{2} \alpha}{\left|\frac{1}{\gamma_{12}} \gamma_{1} \rho_{1}-\frac{1}{2} \gamma_{2} \rho_{2}\right|}+\frac{Z_{2}^{2} \alpha}{\gamma_{2} \rho_{2}},
$$

where $\rho_{1}$ and $\rho_{2}$ are the translation-invariant Jacobi coordinates, $\gamma_{1}$ and $\gamma_{2}$ are the individual parameters (oscillator sizes) for each Jacobi coordinate, in contrast to one such parameter used in the traditional approach, $v_{1}$ and $v_{2}$ are the reduced masses for the introduced Jacobi coordinates, and $v_{12}=v_{1} / v_{2}$. The construction of the translation-invariant and asymmetric wave function of the system and the derivation of the expression for the matrix elements of the Hamiltonian (1) using the Racah technique and Talmi-Moshinsky transformations are thoroughly discussed in Ref. [2]. We should note that, due to the Coulomb character of the interaction between the particles, the calculated energy of the ground state is independent of the spin of the particles. The process of calculating the ground state energy of the considered system consists in a sequence of diagonalizations of the Hamiltonian (1) matrix aimed at finding the values of the parameters $\gamma_{1}$ and $\gamma_{2}$ that minimize the lowest eigenvalue. The approach proposed in Ref. [2] is reduced to the traditional approach by the simple substitution $\gamma=\gamma_{1}=\gamma_{2}$. Then the minimization of the lowest eigenvalue of the matrix of the appropriate Hamiltonian is performed with respect to the unique parameter $\gamma$. The dimension of the matrix is determined by the basis cut-off parameter $N_{\max }$ that allows the inclusion of all the states of the system with the total number of oscillator quanta not exceeding this value.

\section{Results and discussion}

The ground state energies of a number of three-particle Coulomb systems involving up to 28 oscillator quanta have been computed by means of the present method. The algorithm developed in the present work was tested by constructing the variational surfaces of the ground state energy of the considered systems. The characteristic shape of the resulting surfaces is shown in Fig. 1 on the example of the $e^{+} e e$ system. The study of the form of the variational surfaces shows that the considered method is very suitable for the numerical minimization with respect to the nonlinear parameters $\gamma_{1}$ and $\gamma_{2}$, since the obtained surfaces exhibit a global minimum only and no local minima. The results of the calculation of the ground state energy of the considered three-particle systems are summarized in Table 1. The first two columns present the notation of the calculated systems (here the commonly used notation of particles is used) and the ratio of the masses of one of the identical particles and the different one, respectively. The next two columns denoted as $H(\gamma)$ report the minimizing value of the parameter $\gamma$ and the energy of the ground state of the systems calculated using the traditional approach. The next three columns denoted by $H\left(\gamma_{1}, \gamma_{2}\right)$ present the minimizing values of the parameters $\gamma_{1}$ and $\gamma_{2}$, as well as the ground state energy of the systems calculated using the individual parameters for each Jacobi coordinate. The last column presents the "exact" values of the energy and the corresponding references. The dash in the first row of Table 1 for the results of calculation using the traditional approach means that for the epp system the convergence rate of the one-parameter computations was so low that a negative value of the ground state energy could not be obtained up to 28 oscillator quanta. 


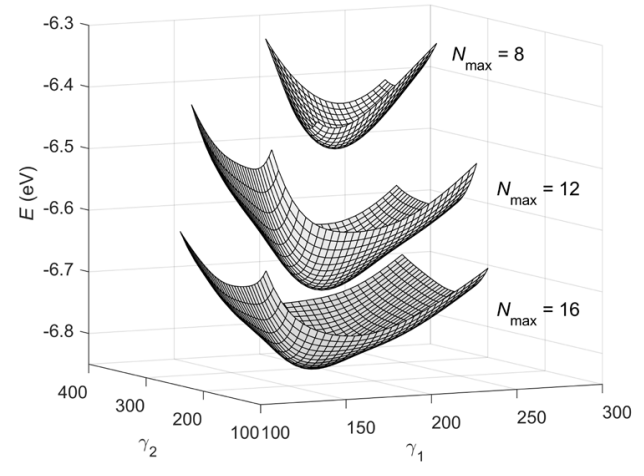

Figure 1. Variational surfaces of the ground state energy of the $e^{+} e e$ system at $N_{\max }=8,12,16$ plotted against the nonlinear parameters $\gamma_{1}$ and $\gamma_{2}$.

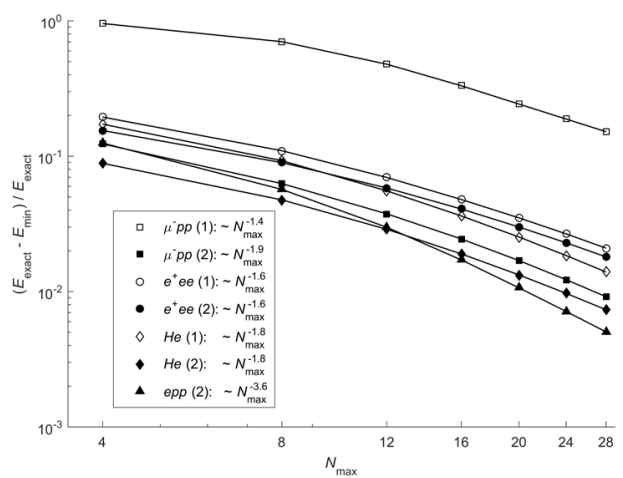

Figure 2. Comparison of the convergence rate for the methods with one general parameter and with individual parameters for Jacobi coordinates in the calculation of three-particle systems $\mu^{-} p p, e^{+} e e$, He, epp.

Results illustrating the computation accuracy and the convergence rate of the traditional method with a single nonlinear parameter and of the method with two nonlinear parameters are reported in Fig. 2 for particular systems. To guide the eye, the symbols which illustrate the dependence on $N_{\max }$ of the outputs of a same method are connected with straight lines. The significantly better performance of the present method is obvious. Following [10], the convergence rate is calculated as the power $q$ of the number of oscillator quanta in the expression

$$
E_{\min }=E_{\text {exact }}+P N_{\max }^{-q},
$$

where $E_{\text {exact }}$ is the result of the "exact" calculation, $P$ is a constant, $E_{\min }$ is the value of the ground state energy of the system, calculated for the given $N_{\max }$. The value of the power $q$ was determined as the slope of the straight line drawn through the last two calculated points in logarithmic scale. The obtained results allow the following conclusions. First, for systems of atomic type (He) the method with two parameters demonstrates much higher precision of the calculations as compared to the singleparameter method, although the convergence rates of both methods in this case are practically the same. Second, for systems with close masses the effect of introducing individual parameters for each Jacobi coordinate is small. The example of the $e^{+} e e$ system shows only a minor increase of the accuracy of calculation in the case of using individual parameters as compared to the traditional approach. The convergence rate in this case is similar for both methods. Third, as shown by the example of the $\mu^{-} p p$ system, with increasing the molecular character of the system, the introduction of individual parameters for Jacobi coordinates substantially improves the accuracy and the convergence rate of the calculations as compared to the traditional approach.

\section{Summary and conclusions}

The harmonic oscillator expansion method using individual parameters for each Jacobi coordinate shows considerable advantage over the traditional approach using only one such parameter in the systems with the masses of two identical particles strongly differing from that of the third particle, as 
Table 1. Ground state energies $E_{\min }$ (in $\mathrm{eV}$ ) for Coulomb three-particle systems with two identical particles calculated for the 28 harmonic oscillator excitation quanta $\left(N_{\max }=28\right)$.

\begin{tabular}{c|c|c|c|c|c|c|c}
\hline & & \multicolumn{2}{|c|}{$H(\gamma)$} & \multicolumn{3}{c|}{$H\left(\gamma_{1}, \gamma_{2}\right)$} & \\
\cline { 3 - 7 } System & $m_{2} / m_{1}$ & $\gamma$ & $E_{\min }$ & $\gamma_{1}$ & $\gamma_{2}$ & $E_{\min }$ & $E_{\text {exact }}$ \\
\hline$e p p$ & $1.8362 \times 10^{3}$ & - & - & 97.7 & $8.70 \times 10^{4}$ & -16.167 & $-16.249[4,5]$ \\
$\mu^{-} p p$ & 8.8802 & 400 & -2359.3 & 118 & $8.33 \times 10^{2}$ & -2756.1 & $-2781.6[4,6]$ \\
$K^{-} p p$ & 1.9006 & 222 & -8845.6 & 148 & 325 & -8962.3 & $-9104.3[4]$ \\
$e^{+} e e$ & 1 & 198 & -6.9803 & 168 & 237 & -7.0008 & $-7.1295[4]$ \\
$p \mu^{-} \mu^{-}$ & $1.1261 \times 10^{-1}$ & 184 & -2585.7 & 227 & 138 & -2604.5 & $-2654.9[4,7]$ \\
$\alpha \mu^{-} \mu^{-}$ & $2.8347 \times 10^{-2}$ & 73.8 & -15658 & 93.7 & 49.3 & -15748 & $-15865[4,7]$ \\
$\mu^{+} e e$ & $4.8363 \times 10^{-3}$ & 185 & -13.854 & 244 & 124 & -14.028 & $-14.288[4,8]$ \\
$K^{+} e e$ & $1.0351 \times 10^{-3}$ & 185 & -13.906 & 247 & 124 & -14.085 & $-14.345[4]$ \\
$p e e$ & $5.4462 \times 10^{-4}$ & 185 & -13.913 & 246 & 124 & -14.092 & $-14.353[4,7]$ \\
$\mathrm{He}$ & $1.3709 \times 10^{-4}$ & 74.2 & -77.900 & 95.4 & 48.0 & -78.423 & $-79.003[4,9]$ \\
$\mathrm{Li}^{+}$ & $7.8208 \times 10^{-5}$ & 46.6 & -195.77 & 59.6 & 30.0 & -196.89 & $-198.08[4]$ \\
\hline
\end{tabular}

well as for small and moderate numbers of oscillator quanta. The latter fact is of particular importance for practical calculations, since in the variational methods the computing time and the computational complexity, as a rule, grow exponentially with the increase of the number of oscillator quanta. We should emphasize the essential advantage of the considered method in the calculation of molecular systems, for which, in the case of strongly expressed molecular character such as epp, the traditional approach is practically inapplicable.

The introduction of a larger number of nonlinear variational parameters complicates the computation procedure. However, the improvement of calculation accuracy allows the solution of an eigenvalue problem with reduced cut-off $N_{\max }$ and, therefore, the computational burden and computing time are reduced, too. The developed method is not restricted to Coulomb systems only. The Coulomb potential can be replaced with or used in combination with practically any pair potential. The developed variational approach with the oscillator basis having individual parameters for each Jacobi coordinate opens new promising potentialities for the solution of cluster models in quantum mechanics.

\section{References}

[1] G.P. Kamuntavičius, J. Math. Phys. 55, 042103 (2014)

[2] A. Deveikis, J. Mod. Phys. 7, 290 (2016)

[3] A. Deveikis, Lith. J. Phys. 57, 66 (2017)

[4] E.Z. Liverts and N. Barnea, Comput. Phys. Commun. 184, 2596 (2013)

[5] M.M. Cassar and G.W.F. Drake, J. Phys. B 37, 2485 (2004)

[6] S. Kilic, J.P. Karr, and L. Hilico, Phys. Rev. A 70, 042506 (2004)

[7] L.U. Ancarani, K.V. Rodriguez, and G. Gasaneo, EPJ Web Conf. 3, 02009 (2010)

[8] A.M. Frolov, J. Phys. B: At. Mol. Opt. Phys. 50, 105102 (2017)

[9] V.I. Korobov, Phys. Rev. A 66, 024501 (2002)

[10] L.M. Delves, Adv. Nucl. Phys. 5, 1 (1972) 